\title{
Event-based surveillance in north-western Ethiopia: experience and lessons learnt in the field
}

\author{
Yumi Toyama, ${ }^{a}$ Masaki Ota ${ }^{a}$ and Belay Bezabih Beyene ${ }^{b}$ \\ Correspondence to Yumi Toyama (email: ytoyama@jatahq.org)
}

This study piloted an event-based surveillance system at the health centre $(\mathrm{HC})$ level in Ethiopia. The system collects rumours in the community and registers them in rumour logbooks to record events of disease outbreaks and public health emergencies. Descriptive analysis was conducted on the events captured at the 59 study HCs in the Amhara Region in north-western Ethiopia between October 2013 and November 2014. A total of 126 rumours were registered at two thirds of the HCs during the study period. The average event reporting time was 3.8 days; response time of the HCs was 0.6 days, resulting in a total response time of 4.4 days. The most commonly reported rumours were measles-related ( $n=90,71 \%$ ). These rumours followed a similar pattern of measles cases reported in the routine surveillance system. The largest proportion of rumours were reported by community members $(n=38,36 \%)$ followed by health post workers ( $n=36,29 \%$ ) who were normally informed by the community members about the rumours. This surveillance system was established along with an existing indicator-based surveillance system and was simple to implement. The implementation cost was minimal, requiring only printing and distribution of rumour logbooks to the HCs and brief orientations to focal persons. In countries where routine surveillance is still weak, an event-based surveillance system similar to this should be considered as a supplementary tool for disease monitoring.

\section{T} he scope of the revised International Health Regulations is not limited to any specific disease but extends to any illness or medical condition irrespective of its origin or source. ${ }^{1}$ This has led to initiatives for event-based surveillance (EBS), which requires rapid detection, reporting, confirmation and assessment of rare and new health events that have the potential to affect public health. ${ }^{2}$ EBS also aims to collect data on events occurring in populations that do not have access to health care through formal channels. Considering the limited access of community members to formal health-care facilities, particularly in rural Ethiopia, ${ }^{3-5}$ the Federal Ministry of Health of Ethiopia distributed rumour logbooks (a register to record information on any outbreaks) to regional, zonal and district health offices as a tool for EBS recording in $2011 .^{6}$ However, the logbooks were rarely used, with only a few events recorded at the district level.
The Japan International Cooperation Agency and the Amhara National Regional State Health Bureau (ANRSHB) implemented a technical cooperation project aiming at strengthening the communicable disease surveillance and response in the Amhara Region of north-western Ethiopia from 2008 to 2015. The project, aiming to strengthen EBS in 2013, expanded the usage of rumour logbooks to health centres (HCs) in 22 study districts. This study describes the experience in implementing EBS at HCs that use rumour logbooks in Ethiopia and reviews its performance between 2013 and 2014.

\section{METHODS}

\section{Study sites}

The Amhara Region is the second most populous region in Ethiopia with a population of 20 million people. The

\footnotetext{
Japan Anti-Tuberculosis Association, Kiyose, Tokyo, Japan and Japan International Cooperation Agency Amhara Region Surveillance and Response Project,

Bahir Dar, Amhara, Ethiopia.

Amhara National Regional State Health Bureau, Bahir Dar, Amhara Region, Ethiopia

Submitted: 11 April 2015; Published: 17 August 2015

doi: 10.5365/wpsar.2015.6.2.002
} 
administrative levels of the region consist of 10 zones with 167 districts and three city jurisdictions. Districts in three zones were selected for the study by convenient sampling upon consultation with the ANRS-HB.

\section{Planning and implementation at the health- centre level}

The project team had two meetings with the ANRS-HB in mid-2013 on how to strengthen EBS in the Amhara Region, particularly on how to record and monitor the involvement of community volunteers in EBS. It was decided that the project would expand the usage of existing rumour logbooks by printing and distributing them to HCs for EBS monitoring. The project started distributing logbooks to $175 \mathrm{HCs}$ in 22 study districts in October 2013.

The project team provided orientations to $\mathrm{HC}$ surveillance focal persons on the use of the rumour logbooks. The surveillance official of the district health office and the project team conducted quarterly monitoring visits to the $\mathrm{HCs}$. $\mathrm{HC}$ focal persons were interviewed to reveal if any unusual health events occurred in the previous quarter. These events were cross-checked with the rumour logbooks. The focal persons were encouraged to use the rumour logbooks if the verbally reported events were not registered in them.

\section{Operational procedures}

\section{Community and health post levels}

In 2012, the Federal Ministry of Health initiated the Health Development Armies (HDAs), a volunteer-based community health team that uses existing human resources in the government health structure to facilitate health promotion within the community. ${ }^{7}$ The team created a network structure of one volunteer per five households $^{7}$ and collected health-related information from these households. For EBS, the community health extension workers (HEWs) instructed HDAs to report any communicable disease outbreaks and unusual health events to health posts (HPs) and HCs, particularly events with multiple deaths from unknown causes. Each HEW who works at a HP serves about 5000 people. HEWs and HDAs were mobilized for verification of the rumours in the community if needed. Their activities were monitored through interviews with the surveillance focal person at
HCs during the quarterly visit by the project and district health officers.

\section{Health centres}

A reported rumour from the community or a HP was registered in the rumour logbook by the surveillance focal person at the HC. Each $\mathrm{HC}$ serves about 25000 people. The focal person reported to the district health office if the rumour was verified. The verification result and the response activities, if applicable, were also recorded in the rumour logbook.

\section{District health offices}

Once a rumour was informed by the $\mathrm{HC}$, surveillance officers at the district health office assisted the $\mathrm{HC}$ with rumour verification, instructed necessary response activities and communicated with zonal and regional health departments for further assistance and logistical supplies. They also monitored the usage of rumour logbooks during quarterly monitoring visits to the HCs.

\section{Data variables}

All variables used in the original rumour logbook by the district or higher level were kept in this study. Variables in the rumour logbook included: (1) epidemic/ event starting date; (2) date a case first appeared at a health facility; (3) date of registration; (4) date a suspected epidemic was investigated by the $\mathrm{HC}$; (5) date the $\mathrm{HC}$ notified a higher level; (6) date the intervention began; (7) date the $\mathrm{HC}$ received a district/ zonal/regional/national response; (8) condition or event; (9) source of rumour; (10) number of cases initially reported for the event; (11) location of the event; (12) result of the investigation; and (13) actions taken.

\section{Data handling and system evaluation}

Registered rumours were collected during $\mathrm{HC}$ monitoring visits. Data were entered into Microsoft Excel 2013, and $95 \%$ confidence intervals for timelines of reporting and response were calculated by the Excel "Confidence" function.

The EBS was evaluated according to its positive predictive value (PPV) of the rumours and timeliness of reporting and response, as well as its acceptability. ${ }^{8,9}$ 
Figure 1. Map of the study districts in the Amhara Region, Ethopia

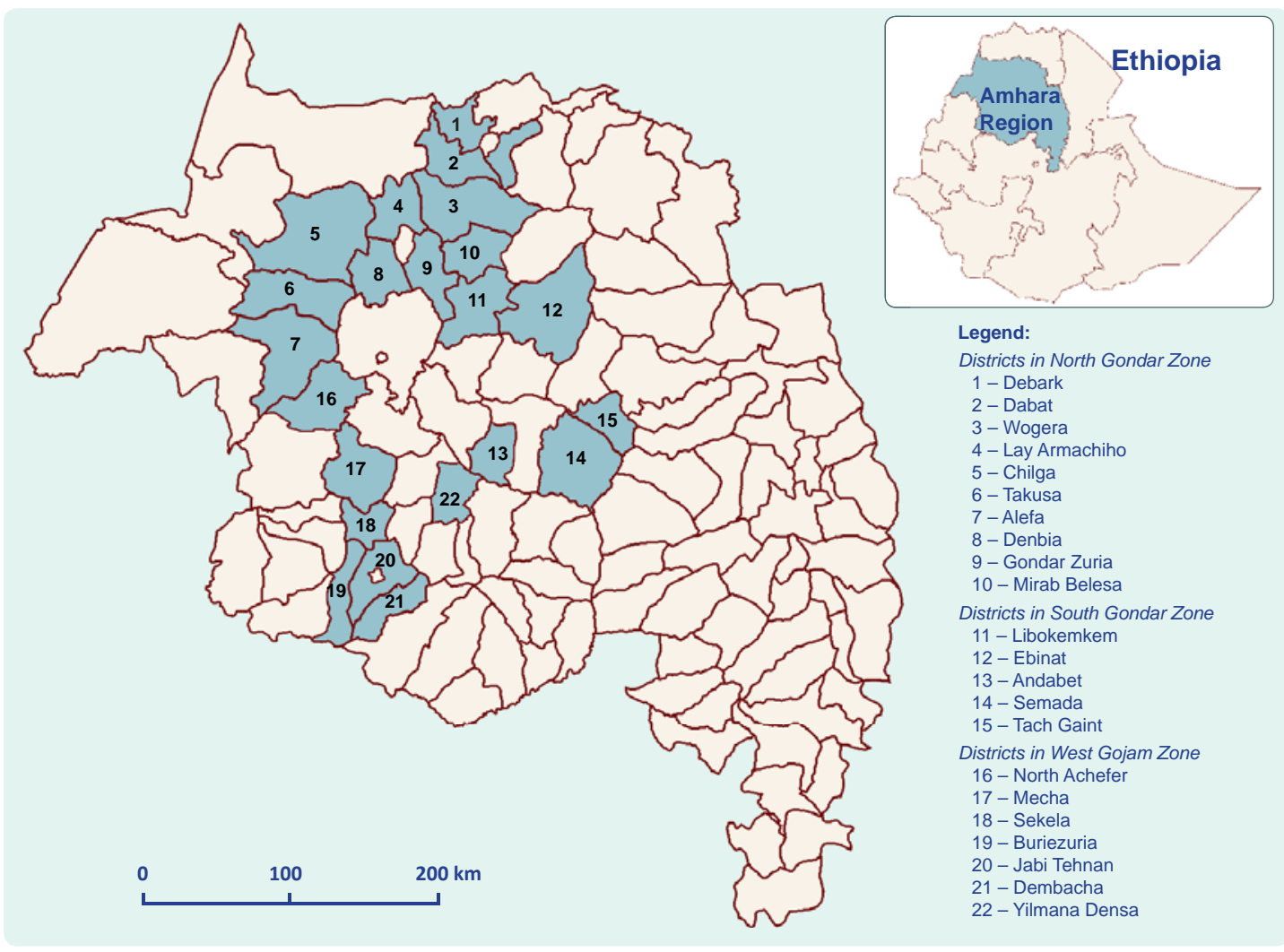

Source: Shape files for the map from ArcGIS (http://www.arcgis.com/home/item.html?id=8296042ed8b14e428491dce57c5c2f7e).

The PPV was defined as the proportion of rumours that were verified as true events among all logged rumours. Acceptability of the system was measured by the proportion of the source of rumours that came from community members among all logged rumours. For timeliness of reporting and response, "reporting time" was defined as the time between the date an event began and the date the event was registered. "Response time of the $\mathrm{HC}$ " was defined as time between the date an event was registered and the date that the $\mathrm{HC}$ started to investigate the suspected event. Timeliness was defined as the relative time frame of reporting time and response time of the HCs. Analysis was limited to the events that all three dates were readily available in the register.

\section{RESULTS}

In total, 22 districts in three zones with an estimated total population of 4.5 million were selected for the study (Figure 1). A total of $59 \mathrm{HCs}$ were selected in these study districts. In the six project pilot districts that were more closely monitored, all of the HCs accessible by car were selected ( $36 \mathrm{HCs}$ ); whereas, in each of the other study districts, only one or two of the HCs that were accessible by car were selected $(23 \mathrm{HCs})$.

The rumour logbooks were available at 41 (69\%) and 54 (92\%) of 59 HCs in May and November 2014, respectively. In total, 126 rumours on outbreaks or public health emergencies were registered at 38 of the 59 study HCs from October 2013 to November 2014. One hundred and nine (87\%) events were reported from six pilot districts. Of the 126 rumours that were reported, 81 (64\%) were verified and found to be true public health events, 16 (13\%) were ruled out and 29 (23\%) did not have records on the results of investigation. The PPV of the rumour surveillance was found to be $0.64(81 / 126)$.

For the 37 (29\%) events that had complete data sets on the reporting time and response time of the $\mathrm{HC}$, the average reporting time was 3.8 days ( $95 \%$ confidence interval $[\mathrm{Cl}]: 2.2-5.3$ ) while the response time of the $\mathrm{HC}$ was 0.6 days $(95 \% \mathrm{Cl}: 0.1-1.2)$, resulting in a total response time of 4.4 days ( $95 \% \mathrm{Cl}: 2.8-6.0$ ). 
Table 1. Distribution of registered rumours and type of responses

\begin{tabular}{|c|c|c|c|c|c|c|}
\hline Events & $\begin{array}{l}\text { Number of } \\
\text { rumours }\end{array}$ & (\%) & Verified & (\%) & Type of action taken* & $(n)^{*}$ \\
\hline \multirow[t]{5}{*}{ Measles } & 90 & 71 & 57 & 63 & Case management & 43 \\
\hline & & & & & Active case finding & 7 \\
\hline & & & & & Vaccination & 2 \\
\hline & & & & & No data & 7 \\
\hline & & & & & Unable to determine & 6 \\
\hline \multirow[t]{2}{*}{ Suspected rabies } & 14 & 11 & 8 & 57 & $\begin{array}{l}\text { Only intervention dates were recorded. } \\
\text { No actual actions listed. }\end{array}$ & 7 \\
\hline & & & & & No data & 1 \\
\hline Anthrax & 5 & 4 & 5 & 100 & Unable to determine & 5 \\
\hline \multirow[t]{2}{*}{ Whooping cough } & 4 & 3 & 3 & 75 & Active case search, case management, vaccination & 1 \\
\hline & & & & & No data & 2 \\
\hline Acute flaccid paralysis/polio & 4 & 3 & 2 & 50 & Case management & 2 \\
\hline Neonatal tetanus & 2 & 2 & 1 & 50 & No data & 1 \\
\hline Being unable to speak & 1 & 1 & 1 & 100 & Patient referred to a hospital & 1 \\
\hline Malnutrition & 1 & 1 & 1 & 100 & Patient referred to a hospital & 1 \\
\hline Meningitis & 1 & 1 & 1 & 100 & No data & 1 \\
\hline Onchoserciasis & 1 & 1 & 1 & 100 & No data & 1 \\
\hline Floods & 1 & 1 & 1 & 100 & No data & 1 \\
\hline Malaria & 1 & 1 & 0 & 0 & No data & 1 \\
\hline Acute watery diarrhoea & 1 & 1 & 0 & 0 & No data & 1 \\
\hline Total & 126 & 100 & 81 & 64 & & \\
\hline
\end{tabular}

* Multiple answers were given.

Table 1 shows the distribution of registered rumours and types of actions taken for each event. The most commonly reported rumours were about measles ( $n=90 / 126,71 \%)$ followed by rabies $(n=14 / 126$, $11 \%)$. Other rumours included anthrax $(n=5)$, whooping cough $(n=3)$ and acute flaccid paralysis/polio $(n=2)$. The most frequent responses to verified measles outbreaks ( $n=57$ ) were case management $(n=43 / 57$, $75 \%$ ) followed by active case findings ( $n=7 / 57,12 \%)$ and vaccination ( $n=2 / 57,4 \%$ ). For suspected rabies outbreaks $(n=8)$, there were no actions listed in seven incidents; in one incident there were no data available for response. Responses of verified anthrax outbreaks were unable to be determined $(n=5)$. The largest proportion of reports came from community members $(n=38 / 126$, $30 \%$ ) followed by HPs workers ( $n=36 / 126,29 \%$ ) who were normally informed by the community members about the rumours. Reports from HCs $(n=26)$, other health facilities $(n=2)$ and schools $(n=2)$ were less common. Twenty-two (17\%) rumours did not have a data source.
The trends of the measles cases reported through the routine indicator-based surveillance system and the rumours registered at the study $\mathrm{HCs}$ were compared (Figure 2). The rumours of measles followed a pattern similar to that of the measles cases reported through the routine surveillance system. For eight verified rumours on rabies/dog bites, only one case was reported as rabies. Five verified anthrax rumours were reported by EBS; however, only two cases were captured in the routine surveillance. For two verified rumours on suspected acute flaccid paralysis/polio and one on suspected neonatal tetanus, none of the cases was captured. Since pertussis was not a reportable disease, for the three rumours on whooping cough, none of the cases was captured in the routine surveillance.

\section{DISCUSSION}

In this study, we described the piloted-EBS and reviewed the rumours on communicable disease outbreaks and unusual events registered in the Amhara Region, 
Figure 2. Measles cases reported through the indicator-based disease surveillance system in the Amhara Region and measles rumours registered in the 22 study districts

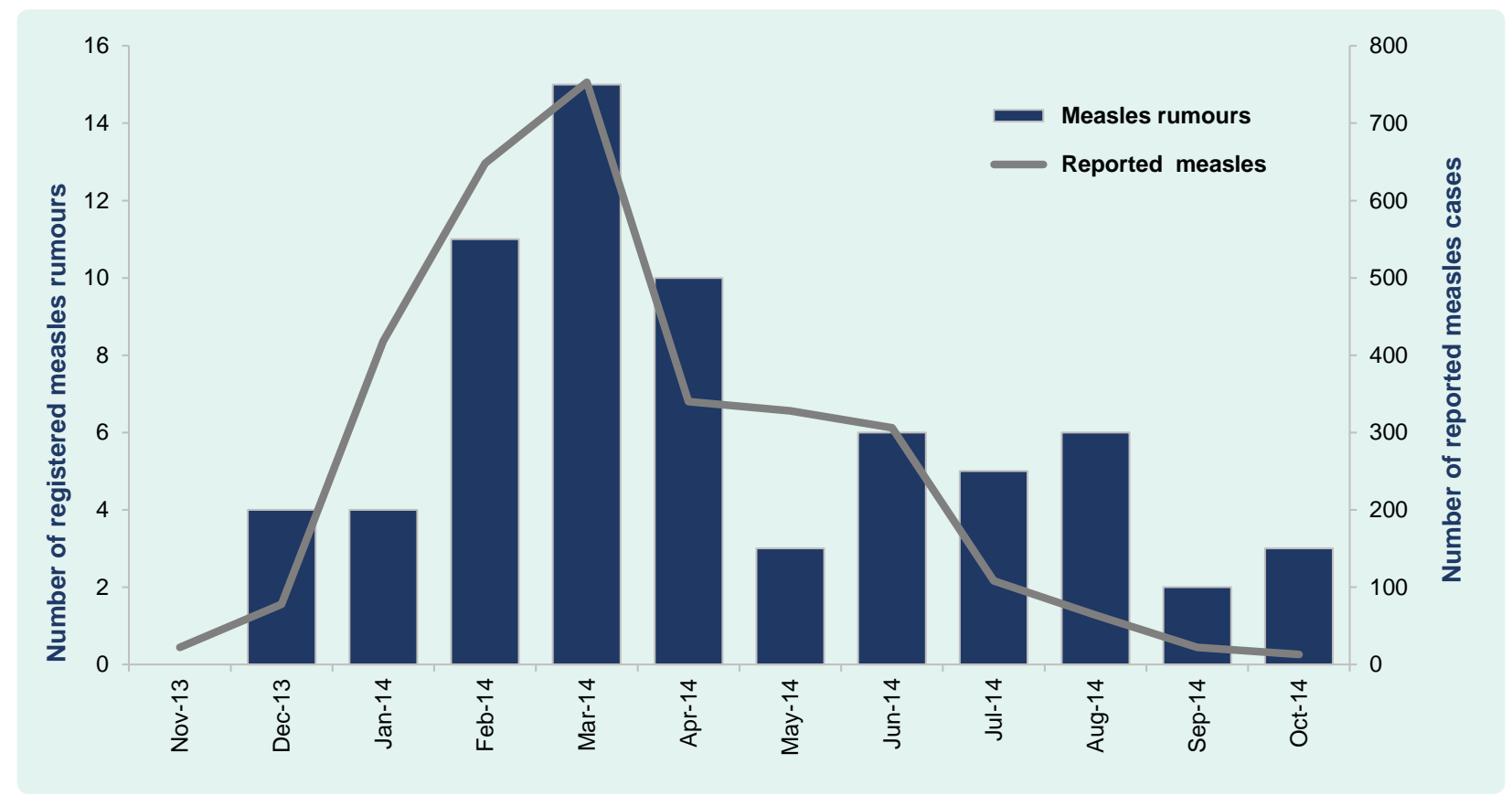

Ethiopia from October 2013 to November 2014. This study is believed to be the first report on EBS at the $\mathrm{HC}$ level collecting rumours from communities in a resource-limited country. This EBS was functional in resource-limited settings like that in Ethiopia, mainly because it was simple to implement and was established along with the existing indicator-based surveillance system. The surveillance focal persons at the HCs had already been trained on disease surveillance in general, including collecting, verifying and responding to rumours if deemed necessary. The cost of establishing the system was minimal, requiring only a brief orientation for the surveillance focal persons and printing and distribution of the rumour logbooks to the HCs. Frequent monitoring visits to the HCs seemed to be effective. The focal persons were encouraged to collect and register the rumours during the visits. The rumour logbooks distributed to $\mathrm{HCs}$ were proven effective to monitor the events.

The acceptability of rumour surveillance is high at the community level, as about one third of the rumours came from community members who were volunteers without any incentives. The average rumour reporting time (3.8 days) in our study was shorter than that in Papua New Guinea (10 days). ${ }^{10}$ This may reflect rumours collection at different levels in the system. While Papua New Guinea collects rumours at the national level, our system collects at the $\mathrm{HC}$ level, which is much closer to the community. Direct outreach to communities for collecting reports was also suggested in the Papua New Guinea EBS system, ${ }^{10}$ which is in line with the findings of this study. Although the reporting time was shorter in Ethiopia than in Papua New Guinea, limited responses to events reported through EBS is a common challenge in both countries. ${ }^{10}$ For example a large number of cases were reported through the EBS during the measles outbreaks in this study; however, vaccination campaigns in only two areas were recorded.

\section{Limitations}

Since the study sites were selected by convenient sampling, the findings presented here may not be representative and generalized for the entire Amhara Region. The performance may be overestimated, as in the six pilot districts, only HCs accessible by car were selected and monitored more closely. Meanwhile, the representativeness of the rumours was depended on how the HDA structure was functioning in the community. Also, the number of HDAs and surveillance focal persons at the HCs who were willing to report and record the rumours, and whether the focal persons knew the correct usage of the rumour logbooks may affect the results. 
Prior training is particularly important to ensure high quality surveillance data.

\section{CONCLUSIONS AND RECOMMENDATIONS}

We recommend an EBS system similar to the one used in this study be implemented at the $\mathrm{HC}$ level with rumour logbooks to monitor the events in countries where routine surveillance is still weak and needs a supplemental tool.

Limited capacity to respond to the rumoured events was found in our study. Thus, the health authorities should strengthen the capacity to provide prompt responses to outbreaks in line with EBS implementation.

\section{Conflicts of interest}

None declared.

\section{Funding}

None.

\section{References}

1. International Health Regulations (2005), 2nd edition. Geneva, World Health Organization, 2008 (http://whqlibdoc.who. int/publications/2008/9789241580410 eng.pdf, accessed 12 March 2015).

2. A guide to establishing event-based surveillance. Manila, World Health Organization Regional Office for the Western Pacific,
2008 (http://www.wpro.who.int/emerging diseases/documents/ eventbasedsurv/en, accessed 12 March 2015).

3. Bilal NK et al. Health extension workers in Ethiopia: improved access and coverage for the rural poor. In: Chuhan-Pole $\mathrm{P}$ and Angwafo $M$, editors. Yes Africa can: success stories from a dynamic continent. Washington DC, The World Bank, 2011: 433-443 (https://openknowledge.worldbank.org/bitstream/handl e/10986/2335/634310PUBOYes0061512B09780821387450. pdf? sequence $=1$, accessed 11 July 2015).

4. Okwaraji YB et al. Effect of geographical access to health facilities on child mortality in rural Ethiopia: a community based cross sectional study. PLOS ONE, 2012, 7:e33564. doi:10.1371/ journal.pone.0033564 pmid:22428070

5. Okwaraji YB et al. The association between travel time to health facilities and childhood vaccine coverage in rural Ethiopia. A community based cross sectional study. BMC Public Health, 2012, 12:476. doi:10.1186/1471-2458-12-476 pmid:22726457

6. Public Health Emergency Management. Guidelines for Ethiopia 2012. Addis Ababa, Ethiopian Health and Nutrition Research Institute, 2012 (http://www.ephi.gov.et/images/guidelines/phemguideline-final.pdf, accessed 12 March 2015).

7. Sibley LM et al. Improving maternal and newborn health care delivery in rural Amhara and Oromiya regions of Ethiopia through the Maternal and Newborn Health in Ethiopia Partnership. Journal of Midwifery \& Women's Health, 2014, 59 Suppl 1:S6-20. doi:10.1111/jmwh.12147 pmid:24588917

8. Crowe $\mathrm{S}$ et al.; Centers for Disease Control and Prevention (CDC). A plan for community event-based surveillance to reduce Ebola transmission - Sierra Leone, 2014-2015. MMWR. Morbidity and Mortality Weekly Report, 2015, 64:70-73. pmid:25632956

9. Updated guidelines for evaluating public health surveillance Systems. MMWR. Morbidity and Mortality Weekly Report, 2001, 50 RR13;1-35. pmid:11215787

10. Dagina R, Murhekar M, Rosewell R, Pavlin BI. Event-based surveillance in Papua New Guinea: strengthening an International Health Regulations (2005) core capacity. Western Pacific Surveillance and Response Journal, 2013, 4(3):19-25. doi:10.5365/wpsar.2013.4.2.001 pmid:24319609 